\title{
Dirección teatral: del mundo del pensamiento al universo de las relaciones de grupo. Reflexiones en torno los procesos de escenificación del teatro independiente de Córdoba
}

Fwala-lo Marin

CONICET, Centro de Investigaciones “María Saleme de Burnichón” FFyH UNC fwalalomarin@gmail.com

Fecha de recepción: 30/08/2018. Fecha de aceptación: 17/09/2018.

\begin{abstract}
Resumen
En el presente trabajo abordaremos los tópicos centrales de diversas fuentes bibliográficas en torno a la noción de dirección teatral. En particular, nuestro enfoque se basa en una perspectiva situada en los procesos de escenificación y en las prácticas del campo teatral independiente de Córdoba y será desde allí que realizaremos la lectura de los escritos. En un segundo momento, como primer acercamiento al concepto específico con el que se opera en dicho campo, se trabajó con un grupo de directores escogidos por sus similitudes de edad y consagración. Parte de las conclusiones de este trabajo describen dos rasgos fundamentales: el primero es que la dirección está unida al mundo del pensamiento y que desde la idea o la imagen mental se activa el proceso creativo. El segundo rasgo es que la dirección implica un trabajo con otros, una capacidad para sostener un "ir juntos", para guiar al grupo y acompañar el proceso de creación conjunto, siendo una actividad eminentemente humana. Estas conclusiones parciales abren un primer panorama, no sólo para conocer el campo del teatro independiente de Córdoba, sino también para arriesgar conceptualizaciones que puedan dialogar con otros territorios.
\end{abstract}

\section{Theatrical Direction: from the World of Thought to the Universe of Group Relations. Reflections on the Staging Processes of the Independent Theatre in Córdoba, Argentina}

\begin{abstract}
In the present work we will address the central topics of various bibliographical sources regarding the notion of theatrical direction. In particular, our perspective is situated in the staging processes and in the practices of the independent theatre field of Córdoba and it will be from there that we will carry out the reading of the writings. In a second moment, as a way to approach the concept as it operates in this specific field, we worked with a group of directors chosen for their similarities of
\end{abstract}

Palabras clave

Estudios Teatrales Dirección Teatral Teatro Independiente Teatro contemporáneo Córdoba, Argentina
Keywords

Theatre Studies Direction Regie

Mise en scène

Independent Theatre Contemporary Theatre Córdoba, Argentina 
age and recognition. Part of the conclusions of this work describes two fundamental features: the first is that the direction is linked to the world of thought and that the idea or the mental image activates the creative process. The second feature is that direction implies a work with others, a capacity to sustain a "going together", to guide the group and accompany the process of joint creation, being an eminently human activity. These partial conclusions open a first panorama, not only to know the field of the independent theater of Córdoba, but also to risk conceptualizations that can dialogue with other territories.

En los siguientes apartados se presentará la cuestión del concepto de dirección teatral. En un primer momento nos proponemos realizar una sistematización del concepto de dirección en el teatro contemporáneo en virtud de fuentes bibliográficas provenientes de enfoques distintos, tanto de estudios europeos contemporáneos (franceses, ingleses, españoles), como de autores argentinos. Una singularidad de estos últimos es que su desarrollo teórico muchas veces está afincado en la producción artística y, en particular, en la dirección. Para organizar los diversos discursos apelaremos a la noción de desacuerdo de Rancière (1996:8) que entiende que se trata de "un tipo determinado de situación de habla: aquella en la que uno de los interlocutores entiende y a la vez no entiende lo que dice el otro". Cabe señalar que las lecturas e interpretaciones se realizan desde la territorialidad del teatro independiente de Córdoba, donde está situada la investigación mayor que enmarca este trabajo. En un segundo momento se abordarán las nociones que presentan distintos directores ${ }^{1}$ durante entrevistas en profundidad, que permitieron observar rasgos comunes aún en poéticas disímiles.

Esta problemática es parte de la investigación "La dirección en el teatro independiente y contemporáneo de Córdoba", realizada en el marco de una beca interna doctoral CONICET, que busca comprender cómo se lleva adelante la dirección en el teatro independiente de Córdoba y aproximarse a una conceptualización vinculada a las prácticas del campo. En dicha la investigación, el interés está en sistematizar esta labor y en conseguir conceptualizarla desde una perspectiva que atienda a los procesos de escenificación y al teatro independiente cordobés, sin desmedro de las relaciones que puedan realizarse con otros territorios. Al mismo tiempo, se espera comprender el sistema de relaciones que opera en la construcción legítima de la definición de dirección teatral que opera en el campo. Nos preguntamos acerca de cuáles son los estilos fundamentales de directores de teatro independiente consagrados de Córdoba nacidos entre 1975 y 1985 y cuál es la noción de dirección con la que llevan adelante su rol. En este artículo, específicamente se atenderá a los avances parciales en torno a dicha noción.

El grupo que conforma la muestra total está seleccionado en función de criterios de consagración y edad ${ }^{2}$, quedando compuesto por doce directores de teatro: David Piccotto, Marcelo Arbach, Daniela Martin, Martín Gaetán, Rodrigo Cuesta, Luciano Delprato, Eugenia Hadandoniou, María Belén Pistone, María Palacios, Gonzalo Marull, Jazmín Sequeira y Maximiliano Gallo. El objetivo de la muestra fue reunir a quienes se encuentren en posiciones cuyo reparto del capital específico las ubique en el centro del campo (Bourdieu 1995: 342; Bourdieu 2013: 31 y Gutiérrez 2012: 42). En un segundo momento se organizó en subgrupos a partir de similitudes en los rasgos estéticos de sus obras. Principalmente se evaluó cuál o cuáles eran los lenguajes con mayor centralidad en la puesta en escena y a qué tipo de materiales/discursos/ textos apelaban los directores. Así es como resultaron cuatro subgrupos que denominamos: Teatro documento, Pensamiento escénico, La Maestría y Los montajistas. El foco de atención en esta etapa de la investigación, y que da lugar a este trabajo, fueron entrevistas a siete directores: David Piccotto, Rodrigo Cuesta, Martín Gaetán, Gonzalo Marull, Jazmín Sequeira, María Belén Pistone y María Palacios. A partir de un
1. Más adelante se presentan los criterios de selección de la muestra.

2. os directores fueron escogidos en función de tres condiciones, la primera es que formen parte del circuito de teatro independiente de la ciudad. La segunda es que hubieran nacido entre 1975 y 1985 . La franja etaria señalada delimita que los sujetos desarrollaron su formación profesional en democracia, vivieron la experiencia de la Crisis del 2001, tuvieron por maestros a personas atravesaron la última dictadura militar como teatristas en actividad, entre otros factores políticos y sociales que podrían marcar sus modos de entender y ejercer la dirección. Y como tercera condición, deben haber sido beneficiados más de una vez en los últimos años por alguno de estas distinciones: participación en la Fiesta Provincial del Teatro (Instituto Nacional del Teatro), Premio a la Producción y Creación Teatral (Gobierno de la Provincia), Fondo Estímulo a la Actividad Teatral Cordobesa (Municipalidad), TEATRES (Municipalidad). A partir de estos criterios se construye una muestra, que luego de ser ajustada considerando algunas alteraciones menores en los sujetos reunidos, se eleva al número de doce directores. 
primer acercamiento mediante entrevistas en profundidad, se pudieron identificar dos rasgos compartidos por este grupo: por un lado, que la dirección opera en el movimiento del mundo del pensamiento al mundo de la escena; y por otro, que la dirección asienta su labor en habilitación de un ecosistema que permita la creación en el marco de un trabajo colectivo.

Para comenzar es necesario situar las actividades sobre las cuales haremos foco en el marco del teatro independiente. Este concepto suele ser huidizo, sin embargo, tomaremos los criterios de investigaciones anteriores sobre el teatro de Córdoba, donde se abordan rasgos particulares que permiten describir la categoría de «teatro independiente» (Halac 2006; Brizuela 2002; Tahan 2000 y Villegas 2000). Además, nos apoyaremos en las definiciones de teatro independiente que están contenidas en la Ley Nacional de Teatro (Ley 24.800 1997). A partir de estos trabajos y en virtud de indagaciones propias, se puede afirmar que bajo la etiqueta de independiente podría agruparse todo el teatro que tiene lugar en la ciudad de marzo a diciembre, producido por grupos autogestivos, apoyados de uno u otro modo por políticas estatales, que se auto-reconocen como independientes en virtud de su poética y sus modos de producción. Las estéticas y las poéticas están fuertemente estructuradas por la búsqueda, la experimentación y los procesos creativos en torno a lo grupal. Ciertas salas son reconocidas como fundamentales en la identidad teatral independiente de la ciudad, en torno a ellas se organizan los agentes. Las salas tendrían el rol de las instituciones en la teoría de campos de Bourdieu (1995). Una de las hipótesis de nuestra investigación es que la experimentación distintiva, junto con los rasgos particulares en las poéticas serían algunas de las reglas de juego para monopolizar el capital específico del campo teatral.

En este trabajo en particular se aborda el grupo de los montajistas, dos miembros del grupo del pensamiento escénico y dos miembros del grupo teatro documento. La aproximación a los directores se llevó adelante mediante entrevistas etnográficas, semiestructuradas. Cada director fue entrevistado en el marco de interrogantes más amplios que los que trataremos aquí, ya que evaluaremos los aspectos pertinentes a este trabajo. Prestaremos especial atención a lo que revelan sobre su modo de ejercer el rol de la dirección y de sus ideas sobre el teatro, el desglose de las actividades que realiza como parte del oficio y, finalmente, a sus propias conceptualizaciones acerca de la dirección.

En relación a aspectos metodológicos, considerando que se realizaron entrevistas, que luego fueron analizadas, se tomaron algunos elementos de Santamarina y Marinas. En su propuesta de modalidad de análisis de las entrevistas distinguen la comprensión escénica. En el modelo de la comprensión escénica el sentido sería producto de la construcción del investigador, en una operación de traducción de lo dicho a otra versión que busca la verdad a través de la interpretación (Kornblit, 2007:18). A la vez, en el relato se actualizan tres tipos de escenas: las vividas en el pasado, las vividas en el presente y las vividas en la entrevista. Las escenas vividas en el presente "configuran el contexto de relaciones actuales del sujeto, de donde también seleccionará aquellas a las que atribuye un sentido relevante" y donde "el acceso a estas escenas no puede ser inmediato ni total sino que está mediatizado por la selección que el hablante realiza, en función de la relevancia que otorga a determinadas interacciones o hitos de su historia" (Kornblit, 2007:18). Este aspecto es particularmente interesante para este trabajo, ya que en el relato de los y las directoras despuntan sólo algunos aspectos centrales de su propia práctica, aquellos a los que dan especial relevancia y emergen fácilmente en su memoria.

Otra cuestión pertinente a la metodología de abordaje de las problemáticas es la condición de particular que como miembro 'artista' del campo a la vez estudio, debo 
considerar. Por ello es que apelo al concepto de etnografía encarnada que propone Aschieri (2018:2) dado que no es posible "suspender el flujo de mis saberes" (1) y realizar

el intento de objetivar al 'sujeto objetivante' [que] tendrá que ver con ubicar al investigador en una posición determinada y analizar las relaciones que mantiene con la realidad que analiza y con los agentes cuyas prácticas investiga así como, con las relaciones que mantiene con sus pares e instituciones (2018:6-7).

De esta forma, considerando que casi todos los miembros del grupo han formado parte de mi trayectoria formativa artística o universitaria, realicé algunas operaciones en los tres momentos que componen el trabajo: el diseño de la entrevista; su realización y luego, su análisis. Para el primer momento, el enfoque fue que los ítems que tocaría la conversación pudieran abarcar la diversidad de las estéticas y los tipos de proceso que ya conocía. Luego de realizar algunas entrevistas, esto fue ajustándose, habilitando a los y las directoras a explayarse en la medida que su práctica ofreciera mayores reflexiones en un ítem o en otro. En la segunda etapa del trabajo relativo a las entrevistas, el esfuerzo fue no dar por sentado aquello que ya conocía de los entrevistados: en el glosario teatral, innumerables términos son opacos, esquivos, por ello iba repreguntando para que sean los entrevistados quienes pusieran en palabras estas nociones. El criterio fundamental fue desnaturalizar aquello que ya conocía. En el tercer momento del trabajo, durante el análisis de las entrevistas, recurrí a categorías de análisis que descompusieran los discursos. Así era más factible, no sólo la comparación, sino también la comprensión de las relaciones entre aspectos de su práctica que parecían inconexos (como, por ejemplo, los objetivos poéticos y los destinatarios esperados). Hasta aquí he presentado los aspectos generales de la investigación y los objetivos propuestos para este trabajo. En el siguiente apartado se analizarán antecedentes y referencias bibliográficas acerca de la dirección teatral.

\section{Antecedentes}

La presentación de las fuentes puede ser comprendida desde la noción de desacuerdo (Rancière, 1996) que toma Boenisch (2015) para abordar los términos directing, mise en scène y regie. Es interesante observar que algunos trabajos se detienen principalmente en la comprensión de los materiales que integran la puesta en escena, mientras que otros se centran en la esfera de la experiencia teatral. Por cierto, Boenisch acude a la palabra «regie», comprendiendo el disenso que se produce cuando alguien dice dirección y otra persona también dice dirección, pero no comprende lo mismo por ese concepto. O bien, no entiende que la otra persona está diciendo la misma cosa en nombre de la dirección (2015:2). Esta noción es ordenadora de los distintos conceptos que se trabajan en este artículo. Los primeros autores que presentaré evocan una concepción más clásica del teatro, a veces rozando el logocentrismo. A continuación, iré ofreciendo una lectura de otros escritos que combinan la comprensión de la puesta en escena como conjunción de lenguajes y la dirección como medium entre la audiencia, el texto escénico y el momento presente (Boenisch, 2015: 21-22).

Es notable que gran parte de la bibliografía específica sobre dirección teatral y puesta en escena proviene de autores que son, a la vez, directores o bien, que además de estas dos identidades son también docentes de espacios universitarios de arte o teatro. Muchas de sus reflexiones son fundamentadas por la práctica en la realización artística o en la enseñanza del teatro. Por ejemplo, Hormigón (2002:27-28), en su libro Trabajo dramatúrgico y puesta en escena recoge su experiencia artística como realizador español y pedagogo para presentar una elaboración de carácter teórica. Allí plantea que el director es el creador del espectáculo y cuenta con la colaboración de un equipo 
que construye elementos de la trama significante y del discurso teatral. Considera que tiene una doble operatividad en tanto es diseñador y conceptualizador, a la vez que se convierte en «inductor» de la creatividad en otros. En otro pasaje se refiere a que la creación de espectáculos es el objetivo de la profesión del director, que realiza "mediante la articulación, selección y organización de los elementos expresivos según criterios estéticos y estilísticos personales, recursos técnicos adecuados y leyes propias de la escena" (132). Con posterioridad afirma que "el director mediante el uso de sus instrumentos de expresión, incluido el trabajo actoral, estableciendo relaciones diversas entre ellos, utilizando métodos y técnicas distintas, enuncia el sentido totalizador de la puesta" (196). De ese modo, define de manera tajante su posición que totaliza al teatro en la esfera del "análisis de los significados (en el sentido semiótico de la interpretación)" y que es "irreconciliablemente opuesta a la atención de la experiencia" (Boenisch, 2015:18).

En un sentido similar, Guillermo Heras (2014), director de escena español y docente universitario, propone inicialmente la comprensión del "director de escena como productor de sentido" (10), que "debe crear una poética propia que configure una dialéctica interna entre las diferentes áreas de creación del proceso escénico y su propia visión del mundo" (18). Heras plantea que hay dos momentos fundamentales en "la construcción de la poética de un espectáculo por parte del director de escena: el tiempo de su estudio personal (...) y el desarrollo de los ensayos" y acentúa la labor con los actores, a los que debe comunicarles su discurso metafórico a los fines de "ser un constructor de mundos sensoriales" (66). Hasta aquí hay un fuerte hincapié en la noción de los sentidos y significados que produce el texto escénico, con una breve mención a la experiencia sensorial. Con posterioridad, Heras plantea la metáfora de un capitán de barco que, tiene una tripulación y unos posibles viajeros para una travesía marítima (77) y, en el mismo sentido, más adelante puntualiza que es "la persona por la que pasan todas las decisiones finales -por muy en equipo o colectivo que sea el trabajo-" (151).

A la vez, en estos y en otros trabajos, nos encontramos con la referencia permanente al término dramaturgia. Danan (2012:12-14), dramaturgo e investigador francés, pone en claro que a los fines de discutir la cuestión de la dramaturgia no es operativa la distinción entre la figura del autor dramático ni del director de escena, sino la función que él denomina dramaturgia. Y propone la definición "movimiento de tránsito de las obras de teatro hasta llegar a la escena" para referirse a la dramaturgia que está (en el territorio del teatro) del lado de la escena y no del texto. En su ensayo atiende a la cuestión de la acción, y retoma a Nancy con "puesta en obra del drama"/"activación de la acción", clarificando al mismo tiempo que la dramaturgia es artificio, fabricación, decisión. Danan acude a las palabras de Dort e introduce la idea de "mentalidad dramatúrgica" para indicar el trabajo que implica imaginar las posibilidades de representación de un texto, responsabilidad que es concerniente a todos los que participan en la creación de un espectáculo teatral "y entre ellos, en primer lugar, el director de escena" (2012:23). El concepto se abre al ampliar la idea de texto por la de material y la de representación, por la de escena. Más adelante, recuperaremos la cuestión de la función dramatúrgica que es tomada por uno de los directores de manera explícita.

Por su parte, Patrice Pavis (2010), en su libro La mise en scène contemporaine, presenta una argumentación en favor de la convergencia de la mise en scène o puesta en escena y de la performance (52), donde distingue la posibilidad de que un teatro como texto y de un teatro como material. En el primer caso, "el director de escena presupone que un texto posee una coherencia que el director encuentra o establece" para construir un espectáculo a modo de ensamble. En el segundo caso, se pierde de vista el objetivo del ensamble armónico y se monta o se conectan los fragmentos verbales y extraverbales 
al interior del espectáculo. Para la primera opción presenta los términos directing a play, mettre en scène, dirigier une pièce, quizá traducibles a dirigir un espectáculo o puesta en escena; mientras que para la segunda opción se refiere a making a performance, fabriquer une représentation, tal vez trasladables a montar una performance. Al respecto, coincidiendo con Jeanmarie Higgins (2014:648-649)

Pavis reconcilia la tendencia posmoderna hacia deconstruir el texto con el deseo del público de "leer" la performance. (...) Pavis desarrolla la idea de que la deconstrucción derrideana desafió las nociones históricas de puesta en escena como la creación de un conjunto estable de significantes, y que a raíz de la supuesta tendencia a la baja de la teoría postestructuralista, la puesta en escena se presenta a sí misma como reconstrucción.

Otra crítica enérgica al teórico francés viene de Peter Boenisch (2015), en su libro Directing scenes and sense, en el que tensiona la noción de teatro que es definido solamente como una "máquina semiótica de representación de significados, transportando la página escrita, a través del escenario, a la audiencia que tiene que decodificar su mensaje en la forma correcta" advirtiendo que el otro extremo tampoco es deseable ya que con una postura polarizada de ese modo "nos perdemos tanto como si fantaseamos con la performance teatral como una experiencia fenomenal puramente presemántica" (9). En este sentido, el autor británico propone una mediación diferente a aquella que ocurre de la página al escenario para entender la dirección como un proceso de mediación donde se organizan las escenas y los sentidos que interconectan procesos de dirección (y performance) con actos de espectación (21-22). El autor plantea que el verdadero sentido de la producción teatral emerge "de la quintaesencia del encuentro del público, facilitado por la dirección y sus relaciones reflexivas: un encuentro con tradiciones culturales y registros de la memoria cultural, pero últimamente también el encuentro con nosotros mismos como espectadores" (22). Nuestra hipótesis es semejante a esta propuesta, ya que en sus relatos algunos de los directores explicitan su rol como mediadores entre el texto escénico y los espectadores.

En otro orden de ideas, volviendo sobre otras fuentes propias del teatro argentino, los textos que siguen provienen del campo teórico de la teatrología y también, al campo de las escrituras y sistematizaciones de teatristas, incluyendo los textos que se sitúan entre estas dos escrituras. El caso del director Ricardo Bartís (2003) en vínculo con el investigador Jorge Dubatti, es ejemplar. Sobre la práctica directorial afirma

el director es el responsable de la combinatoria de todas las fuerzas o elementos de aquello que se producen en el escenario. Es el que intenta generar el ritmo (...) como idea teatral, las combinatorias entre el tiempo y el espacio, y sobre todo las relaciones de lenguaje de la actuación. Por otro lado formula o plantea un campo de ideas (...) y propone tomar caminos laterales, indirectos, multiplicar esas ideas (...) pero por sobre todo producir materialidad escénica, temperatura, gestos, ritmos que se van seleccionando (Bartís, 2003:115).

De esta manera, el autor plantea un campo de acción al interior del texto escénico, donde es posible imaginar que los sentidos que pretende multiplicar involucran a un espectador activo. Dubatti (2003:7) conceptualiza que la noción de teatro que moviliza la labor de Bartis se funda en un teatro como experiencia efímera que "acontece en los cuerpos de los actores, en el convivio y el encuentro de presencias. Bartís asimila la percepción teatral a la de devenir de nuestro régimen de experiencia real". De este modo, la poética de su teatro integra la noción de medium que revisamos con anterioridad.

Con otras concepciones sobre las instancias que general el acontecimiento teatral (Dubatti, 2012:33), pero considerando a la dirección como ligada a la puesta en escena 
en el presente, podemos leer los planteamientos de Rubén Szchmacher (2015), director de teatro. Él considera que la dirección implica el nacimiento simultáneo del objeto y la acción: "La dirección es una acción, un conjunto de operaciones sobre la materialidad de los elementos propiamente teatrales, que posibilita que, de manera más o menos eficaz, se constituya la puesta en escena" (2015:13). El autor recoge sus experiencias como director en, donde desglosa varios aspectos en los que se asienta la dirección: las decisiones sobre la puesta en escena, en primer lugar (97); su dependencia inevitable a los demás miembros del proyecto porque la palabra del director está mediatizada por las de los demás (115); la responsabilidad que tiene para generar relaciones entre los espectadores y la puesta en escena (131).

En contraste a esta concepción del trabajo colectivo, Víctor Arrojo (2014), director y pedagogo mendocino plantea un enfoque diferente a los anteriores al referirse a la cuestión grupal y los roles de la dirección, haciendo hincapié en el aspecto motivacional del director en el proceso de creación. Entiende que las funciones principales son la estimulación, la observación crítica, la elaboración y selección de recursos expresivos, la consideración del espectador, la capitalización de la etapa de funciones.

Es relevante considerar la propuesta de Cipriano Argüello Pitt (2015), director y docente universitario cordobés, que considera que "la pregunta sobre qué es un director de escena es la pregunta sobre el teatro" (14) y plantea como hipótesis que "el realismo funda la tradición de directores dramaturgos". Considera que el ensayo es decisivo en relación a las preguntas que plantea, ya que una metodología u otra alumbrarían una definición de dirección y, de teatro. Su conceptualización se afianza en el territorio del ensayo, ya que considera que ese "es el campo de trabajo del director, que debe estimular (...), coordinar las fuerzas individuales y colectivas en la creación de una tercera cosa: la obra" (82). Su labor es propiciar las condiciones para que sucedan «cosas», hallazgos (83). Además de la cuestión del ensayo, presenta como actividad de la dirección la organización de los materiales, "creación composición y montaje (...) mi perspectiva es la de un director que crea una dramaturgia compleja de la escena". El autor dialoga con las nociones de Danan (2012) que tomamos en este trabajo para manifestar su postura acerca de que el director, con su técnica ya no busca transmitir mensajes, sino que "lo que se pone en relieve el juego por el juego mismo; el placer de conocer sobre el sentido de lo que se conoce" y "es una mirada sobre el procedimiento, que permite una discusión sobre los estatutos de la representación" (33-34).

De esta forma, entre la dicotomía de puesta en escena como máquina semiótica y acontecimiento teatral como pura experiencia van abriéndose las nociones de teatro que movilizan modos de llevar adelante la dirección y modos de conceptualizarla.

\section{Surgimiento}

Sobre la cuestión del surgimiento del director, Pavis, en su Diccionario del Teatro, plantea que "la aparición de la función y del término suele situarse en la primera mitad del siglo XIX" y que es la "persona encargada de montar una obra, asumiendo la responsabilidad estética y organizativa del espectáculo, eligiendo los actores, interpretando el sentido del texto, utilizando las posibilidades escénicas puestas a su disposición" (2011:134).

Sobre el origen del rol, vale la pena tener en cuenta la sistematización de Proust (2001:471) donde fija la atención en la emergencia del teatro de arte en Rusia, Italia, Alemania, Francia e Inglaterra, donde la puesta en escena comienza a constituirse 
como disciplina autónoma, lo que implica una nueva figura la del director de escena que se reivindica como "el intérprete central de la obra y se plantea obligatoriamente en un segundo escritor" (Abirached, 1992:165). Ubica como hitos significativos

una serie de rupturas estéticas, con el surgimiento de nuevas corrientes como el naturalismo, pero también éticas ( ), genera preguntas sobre la puesta en escena, mientras que en la división del trabajo artístico, se define el puesto de director. (Proust, 2012:96)

En el mismo artículo precisa una serie de características sobre el rol de la dirección que ofrecen otras aristas a la función dramatúrgica y el proceso creativo. Se destaca la cuestión ontológica en relación a la inmaterialidad de la labor del director

La representación teatral se basa en elementos materiales: el texto, los cuerpos de los actores, los decorados, etc. Pero estos elementos no hacen una puesta en escena. Esto es reclamado y reconocido como una obra de arte por su capacidad de vincular estos elementos, combinarlos y subsumirlos en una nueva realidad profundamente inmaterial que descansa, en su totalidad y unicidad, en ningún soporte material. (Proust, 2012:103)

El autor basa sus investigaciones en el teatro público francés (estatal), punto culmine de una carrera de director exitosa, que comienza en una compañía independiente de escasos recursos. El avance de una trayectoria en este sentido está condicionado por el paso de una serie de pruebas "que demuestran el dominio de las diversas dimensiones de la actividad del director", y con esto refiere a que

\begin{abstract}
Al dirigir todas las estructuras de producción del teatro público, el director es responsable de las tareas administrativas y la coherencia general de las representaciones para las que es generador "de unidad, cohesión interna y dinamismo, representación" (Roubine, 1980, p 40). Este trabajo de coordinación es inseparable de la afirmación de una doble postura de artista e intelectual. () En una producción del teatro público, el director es, por lo tanto, un artista al que se reconoce tanto la intencionalidad como el dominio de toda la producción de una obra. (Proust, 2012:106)
\end{abstract}

De esta manera, se puntualiza que la función dramatúrgica debe darse en simultáneo a las habilidades de gestión y administración y que, a la vez, el rol involucra un aspecto de desempeño intelectual en el mundo público. Todo ello lo coloca "en el centro de las interacciones del trabajo".

\title{
La dirección en el teatro independiente argentino: el caso de Córdoba
}

En este apartado presentaremos los discursos de los directores, para elaborar algunos tópicos que resuenan con los antecedentes que desarrollamos anteriormente. Anticipamos que las posturas que consideran la puesta en escena exclusivamente en términos de significaciones están ausentes. En un sentido opuesto a los antecedentes sintetizados, la concepción de grupo/colectivo creativo tiene una gran importancia y forma parte del núcleo de la conceptualización a la que arribamos. Al realizar la aproximación a los directores, se develó en sus relatos una conceptualización del objeto de la dirección, una descripción de las prácticas que la constituyen y cuáles son aquellas posturas/tareas/acciones que no deberían formar parte del rol.

En este trabajo recogemos aquellos puntos de acuerdo entre los directores que forman parte del estudio, que, a pesar de las grandes diferencias entre sus poéticas, 
presentan rasgos comunes: disposiciones que los llevan a imaginar obras teatrales, a ensayarlas, a ponerlas en escena, sus modos de ensayo, sus maneras de valorar aspectos del proceso creativo o de la circulación de la obra por sobre otros, a partir de sus concepciones sobre el teatro y la dirección.

\section{¿Qué hace?: tareas del director}

Es esclarecedor tener en cuenta es la descripción que ellos realizan sobre sus prácticas, que coinciden en las siguientes etapas:

a) su responsabilidad en relación a los grupos. En sus palabras: “impulsar al equipo, armonizar, sumar y rearmar el equipo" (Martín Gaetán); "guiar al grupo, sostener la confianza, contenerlo" (Rodrigo Cuesta); "escuchar el trabajo de los miembros y armonizar al grupo" (David Piccotto); "soñar con equipos de trabajo, igualar en ese sueño [creación de una obra y sus procesos de investigación correspondiente] con el equipo de trabajo" (Gonzalo Marull); "armar un ecosistema [creativo] potable (...) encauzar las tensiones y que convivan" (María Palacios); "escuchar, preguntar [al grupo], abrir la comunicación para que circule y que la discusión sea realmente colectiva" (Jazmín Sequeira). Con algunos matices entre ellos (la idea de armonizar difiere de la igualar sueños), asumen un rol fundamental en la construcción de la colectividad.

b) Decisiones y planificación del proceso creativo/de trabajo. En sus palabras: "decide acerca de las limpiezas de escenas, "pelea" con las otras áreas para la condensación" (Gaetán); "tomar decisiones, decidir lo que funciona” (Rodrigo Cuesta); "organizador de los puntos de atención del espectador, decidir qué filtrar desde afuera hacia adentro del grupo" (David Piccotto); "decisión de las totalidades, junto a los técnicos también, los artistas que acompañan al director" (Gonzalo Marull); "observación, escucha y toma de decisiones. Primero se diseña el dispositivo, después se lleva a escena a esos cuerpos, se observa, se escucha (...) y se toman decisiones en relación al funcionamiento del dispositivo" (Belén Pistone); "montar, el trabajo de montaje constante" (Jazmín Sequeira). Asumen que las decisiones, si bien se construyen colectivamente, en el respeto por las responsabilidades de los roles, aun en la desjerarquización de estos, implica una palabra última por las definiciones que constituyen la puesta en escena. Al respecto Gonzalo Marull puntualiza que si bien no acuerda con el lugar totalitario para la toma de decisiones, los miembros de los grupos reclaman al director ese lugar. Jazmín Sequeira propone un sitio desjerarquizado de la dirección buscando precisar su especificidad.

Entre otras tareas a realizar que no fueron comunes por la totalidad de los directores sino por cuatro de ellos (Martín Gaetán, Gonzalo Marull, Belén Pistone y María Palacios) son:

c) Investigar para el proceso de creación. En sus palabras: "Investigar teóricamente lo que ocurre en el ensayo (temática, procedimiento, etc)" (Martín Gaetán), "la primera [tarea] es apasionarme con alguna materialidad textual. La segunda sería un trabajo de investigación sobre el autor, el tema" (Gonzalo Marull). Se podría intuir que hay un trabajo en solitario de la dirección respecto del "universo perceptivo", en palabras de María Palacios.

En relación a la actuación y a las exigencias sobre los actores, todos los directores plantean un proceso de trabajo intenso, ya sea de mayor o menor duración. Los objetivos que se proponen y el correspondiente modo de abordarlo presentan algunos 
matices. Dos de los directores, Rodrigo Cuesta y Martín Gaetán, manifestaron que llevaron adelante un entrenamiento intenso con un grupo de actores, y que continuaron trabajando con ellos en un código y disciplina de trabajo compartidas. Dado que sus elencos suelen ser los mismos, ese tipo de proceso se realizó (casi) por única vez. Cercana a esta metodología, Belén Pistone trabaja minuciosamente con los actores para "encontrar el tono" de la escena. Otra versión del trabajo intenso y sostenido viene de la mano de Jazmín Sequeira, que a partir de un objetivo de indagación/ experimentación específico toma largos periodos de tiempo para entrenar hasta que la obra "florece". Por el contrario, Gonzalo Marull y David Piccotto, explicitaron que convocan actores, pero no pretenden un entrenamiento, ya que "el trabajo es el entrenamiento" que aborda el universo poético de la obra (Gonzalo Marull): de todas formas, el proceso incluye un trabajo de ensayos que busca poner a punto la actuación con los demás elementos de la escena. María Palacios, en un sentido similar plantea un trabajo de entrenamiento/caldeamiento que acerque al universo perceptivo de cada espectáculo en particular.

En relación al acontecimiento teatral, con un acontecimiento que reúne el convivio, el acontecimiento poético y el acontecimiento espectatorial (Dubatti, 2012), no todos ellos se detuvieron en precisar cuestiones concernientes al vínculo con el espectador. Si bien tácitamente suponían que es un factor obvio, dos directoras precisaron su propia definición de la dirección fuertemente ligada a la idea de encuentro: María Belén Pistone se refiere: "la palabra anfitrionazgo es lo que signa mi trabajo. [¿Qué es la dirección?]Pergeñar un encuentro, ser el anfitrión, es un anfitrionazgo", donde su quehacer es propiciar las condiciones para el disfrute de unos otros, y el accionar de aquellos que tienen un rol eminentemente activo durante la función. Jazmín Sequeira, por su parte se refirió a

un rol de habilitación de potencias colectivas que deviene en obra poética. Tiene que ver con habilitar el encuentro y habilitar teniendo presente, como los deseos que arrastran potencias y que contagian, que permiten circular y amplificar y multiplicar lo que podemos. Lo que podemos. Es pensar, es un rol que por lo menos en mi, trata de pensar cómo habilitar lo que podemos juntos y juntas, una potencia vital.

La idea de preparar y disponer un encuentro entre los miembros del equipo creativo, en primera instancia, pero luego, de esos miembros, la poética creada y los asistentes a la experiencia teatral: podría arriesgarme a decir que ese es el concepto con el que operan los directores y las directoras de este grupo. A modo de comentario, expresaron espontáneamente qué no debería hacer un director, como, por ejemplo "quedarse en las búsquedas conservadoras, asumir un lugar subsidiario de las políticas estatales"; "juzgar al actor después de las funciones, esperar resultados perfectos, no cuidar al actor". Dos de ellos, David Piccotto y Gonzalo Marull observan como negativa la tarea del director como productor ejecutivo, organizando los recursos materiales, el tiempo, las personas y la prensa. Por su parte, Gonzalo Marull, explicitó que la dirección no puede conceptualizarse como dirección de actores, ya que eso reduce el universo de trabajo del director ya que "el actor es también en un contexto, en un espacio, en un tiempo".

\section{Conclusiones}

Parte de las conclusiones de este trabajo describen dos rasgos fundamentales, el primero es que la dirección está unida al mundo del pensamiento y que desde la idea o la imagen mental se activa el proceso creativo. Consideramos aquí el concepto de dramaturgia en el sentido 2 y el de mentalidad dramatúrgica propuesto por Danan 
(2012) que revisamos con anterioridad. Por ello, nos parece pertinente transcribir un pasaje de la entrevista a Gonzalo Marull, quien toma estos conceptos desde la idea del director en su función dramatúrgica

Salí en búsqueda de lo que se puede considerar la dramaturgia pero encontré la función dramatúrgica, más que el rol del dramaturgo. ( ) Me encontré con docentes que escribían y dirigían. Esos dos caminos son componer u organizar una obra de teatro, pero también, pensar y reflexionar sobre el traspaso, el traslado, la transferencia de eso a la escena. Aprendíamos de los códigos de la escena.

Si con anterioridad pudimos esclarecer que la dirección tiene a su cargo decidir (en solitario o con otros) entonces, la cuestión de la dramaturgia como tránsito a la escena (tránsito del material que sea) es una responsabilidad del director y allí quedaría circunscripta buena parte de su labor inmaterial.

El segundo rasgo es que la dirección implica un trabajo con otros, una capacidad para sostener un "ir juntos", para guiar al grupo y acompañar el proceso de creación conjunto, siendo una actividad eminentemente humana. Retomaremos las palabras de Marull que, buscando un paralelismo con el director de una orquesta y su tarea aparentemente simple de agitar la batuta, develó poéticamente las claves sobre este aspecto del rol

Lo que yo creo es que el trabajo del director implica el extraer de la composición, por decir, la musicalidad que pasa por tu brazo, que llega al instrumento del músico, eso tiene que ingresar al interior del músico y tu tarea es sacar del interior de él, de esa alma, de esa sonoridad, que si vos no estuvieras no la podría sacar solo. Entonces tenés que extraer desde adentro esa sonoridad para que llegue a [el espectador]. No es un movimiento, no es vos parate acá, 'hoy vamos a ensayar así, vos te vas a parar acá', no. Es un trabajo que implica algo a veces innombrable que tiene que ver con algo que no se puede listar. Creo que la gran tarea del director es esa, que uno planifica todo para eso, para lograr en algún ensayo decir 'logramos sacar del interior de los actores esto y ahora vamos a repetirlo'.

En síntesis, el trabajo del director de teatro independiente en Argentina, y en Córdoba en particular, está vinculada a guiar a la grupalidad, al colectivo, en busca de poner en escena una dramaturgia específica. Esta dramaturgia se construye colectivamente, pero es el director quien asume, o a quién le delegan, la última palabra en las decisiones a tomar. 


\section{Bibliografía}

» Argüello Pitt, C. (2015). Dramaturgia de la dirección de escena. México: Paso de Gato.

》Arrojo, V. (2014). El director teatral es o se hace. Buenos Aires: Editorial INT.

" Aschieri, P. (2018) "Hacia una etnografía encarnada: la corporalidad del etnógrafo/a como dato en la investigación". En Guilhon Antunes Camargo, G., Antropologia da Dança IV. (pp. 74-104). Florianópolis: Insular.

»Bartís, R. (2003). Cancha con niebla. Buenos Aires: Atuel.

"Boenisch, P. (2015). Directing scenes and senses: The thinking of Regie. Manchester University Press.

»Bogart, A. (2013). La preparación del director. Siete ensayos sobre teatro y arte. Barcelona: Alba.

» Bourdieu, P. (1995). Las Reglas del Arte. (Sexta ed.). (T. Kauf, Trad.) Barcelona: Anagrama.

"Bourdieu, P. (2013) El sentido social del gusto. Elementos para una sociología de la cultura. Buenos Aires: Siglo Veintiuno Editores.

» Cosentino, O., Gené, J. C., Pavlovsky, E., Erguza, T., Kartun, M., Bartís, R., Sagaseta, E. (2010). La Puesta en Escena en el teatro argentino del bicentenario. Buenos Aires: Fondo Nacional de las Artes.

»Danan, J. (2012). Qué es la dramaturgia y otros ensayos. México DF: Paso de Gato.-

»Dubatti, J. (2012). Introducción a los estudios teatrales. Buenos Aires: Atuel.

»Dubatti, J. (2013) “Teatro Perdido". En Bartis, R., Cancha con niebla. (pp.7-8). Buenos Aires: Atuel.

"Dubatti, J. (2016) Una Filosofía del teatro. El teatro de los muertos. Lima: Ensad.

"Fliotsos, A., \& Vierow, W. (2013). International Women Stage Directors [Mujeres directoras de escena. Casos internacionales]. Illinois: University of Illinois Press.

»Gutiérrez, A. (2012). Las prácticas sociales: una introducción a Pierre Bourdieu. Villa María: Eduvim.

» Halac, G. (2006). Teatro independiente de Córdoba. Identidad y memoria. Buenos Aires: Editorial del Instituto Nacional del Teatro.

» Heras, G. (2014). Los retos de la Dirección de Escena actual. León, México: Luciérnaga Azul.

» Higgins, J. (2014). Contemporary Mise En Scène: Staging Theatre Today by Patrice Pavis (review). Theatre Journal 66(4), 648-649. Johns Hopkins University Press. Retrieved August 29, 2018, from Project MUSE database. [en línea]. Consultado el 20 de julio de 2018 http://muse.jhu.edu/article/5639955

» Hormigón, J. A. (2002). Trabajo dramatúrgico y puesta en escena (Vol. I). Madrid: Publicaciones de la Asociación de Directores de Escena de España.

» Pavis, P. (2010). La mise en scène contemporaine. Origines, tendances, perspectives. Paris: Armand Colin. 
» Pavis, P. (2011). Diccionario del teatro. Dramaturgia, estética, semiología. Buenos Aires: Paidós.

»Pavis, P. (2016). Diccionario de la performance y del teatro contemporáneo. Ciudad de México: Paso de Gato.

»Proust, S. (2001). Une nouvelle figure de l'artiste : Le metteur en scène de théâtre [Una nueva figura artística: el director de escena de teatro]. Sociologie $\mathrm{Du}$ Travail, 43(4), 471-489. Obtenido de http://www.jstor.org/stable/41928751

»Proust, S. (2012). Les metteurs en scène de théâtre entre réussite sociale et remise en cause ontologique [Los directores de escena teatral entre el exito social y el cuestionamiento ontológico]. En N. Heinich, \& R. Shapiro, De l'artification. Enquêtes sur le passage à l'art (págs. 95-112). Paris: Éditions de l'École des hautes études en sciences sociales.

"Rancière, J. (1996). El desacuerdo. Política y filosofía. Buenos Aires: Ediciones Nueva Visión.

» Szuchmacher, R. (2015). Lo incapturable. Puesta en escena y dirección teatral. Buenos Aires: Reservoir Books.

» Tahan, H. (2000). Teatro Argentino. Escenas interiores. Bs As: Instituto Nacional del teatro/Artes del Sur. 